\title{
Rational Design of Single-Atom Catalysts for Enhanced Electrocatalytic Nitrogen Reduction Reaction
}

Sakshi Agarwal ${ }^{\dagger}$, Ritesh Kumar ${ }^{\dagger}$, Rakesh Arya $^{\dagger}$, and Abhishek K. Singh ${ }^{\dagger *}$

$\dagger$ Materials Research Centre, Indian Institute of Science, Bangalore 560012, India

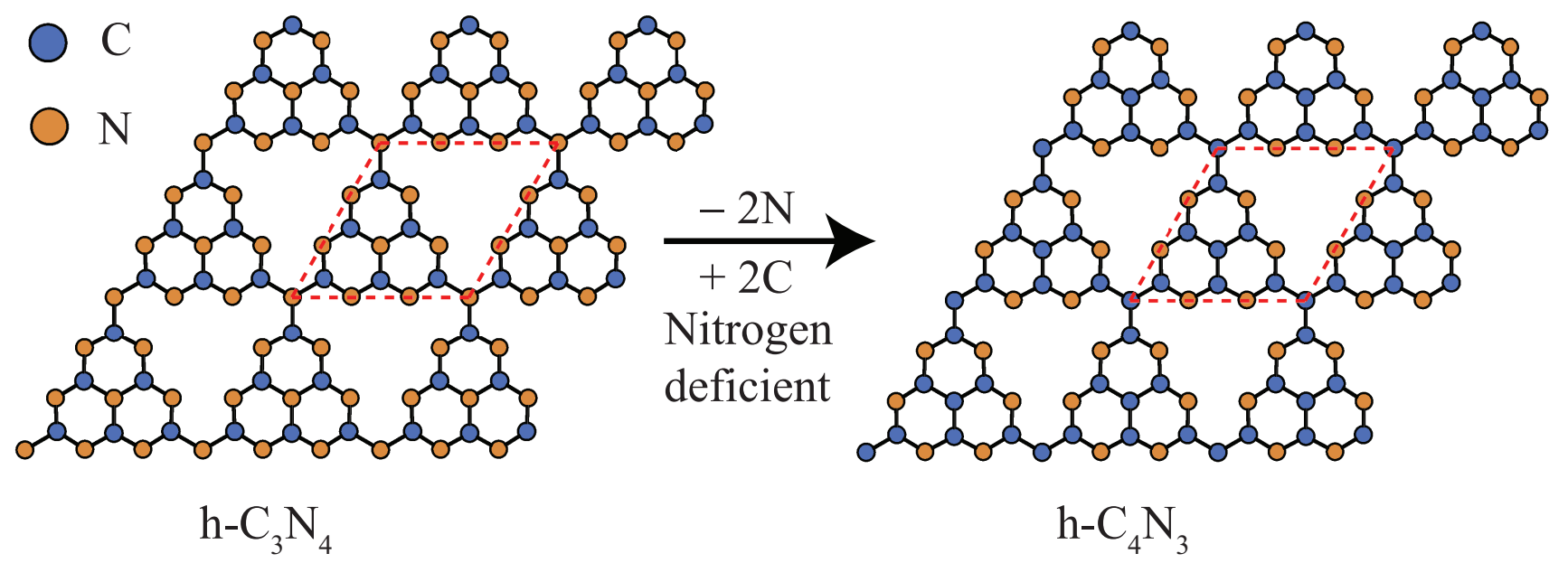

Figure S1: Structures of $h-C_{3} N_{4}$ and h- $C_{4} N_{3}$. With the substitution of $2 \mathrm{~N}$ with $2 \mathrm{C}$, the $\mathrm{h}-\mathrm{C}_{4} \mathrm{~N}_{3}$ is formed 

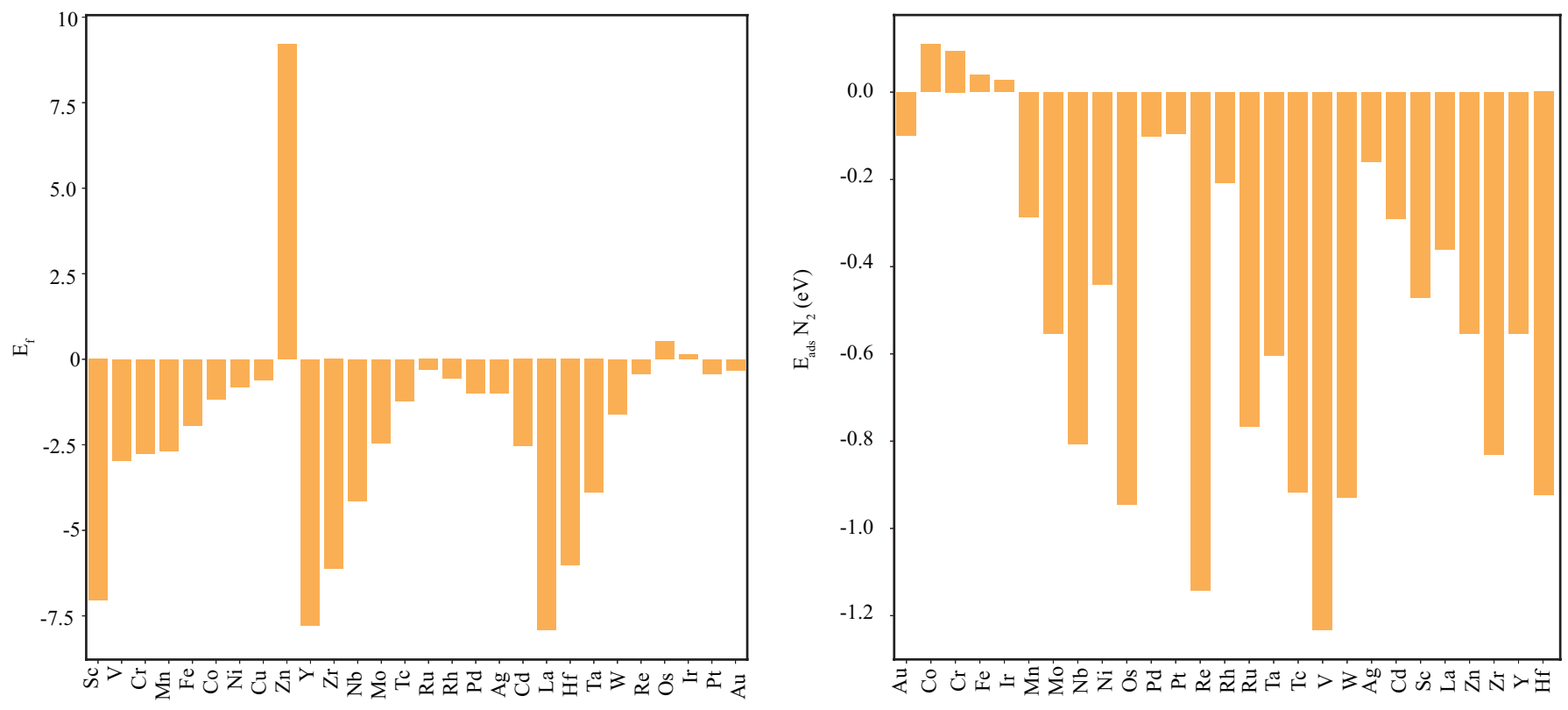

Figure S2: (a) The formation energies $\left(\Delta \mathrm{E}_{f}\right)$ for all the SACs. (b) The adsorption energies $\left(\Delta \mathrm{E}_{a d s}^{N_{2}}\right)$ for $\mathrm{N}_{2}$ adsorption on all the SACs 
(a)

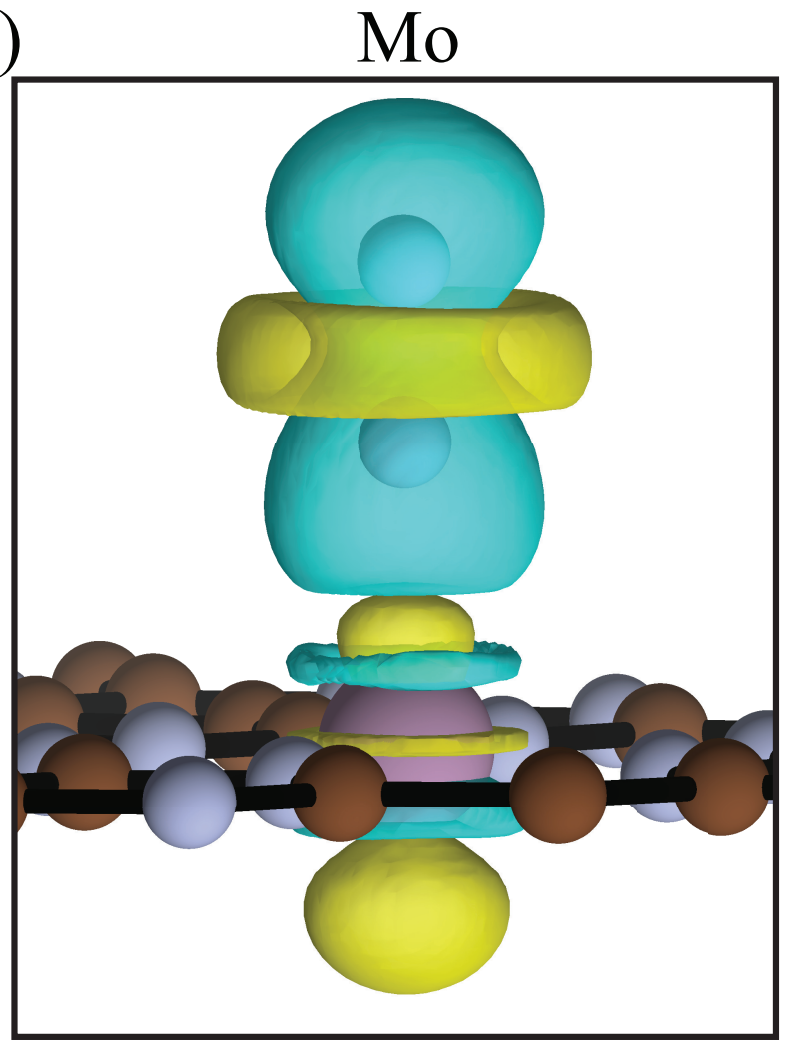

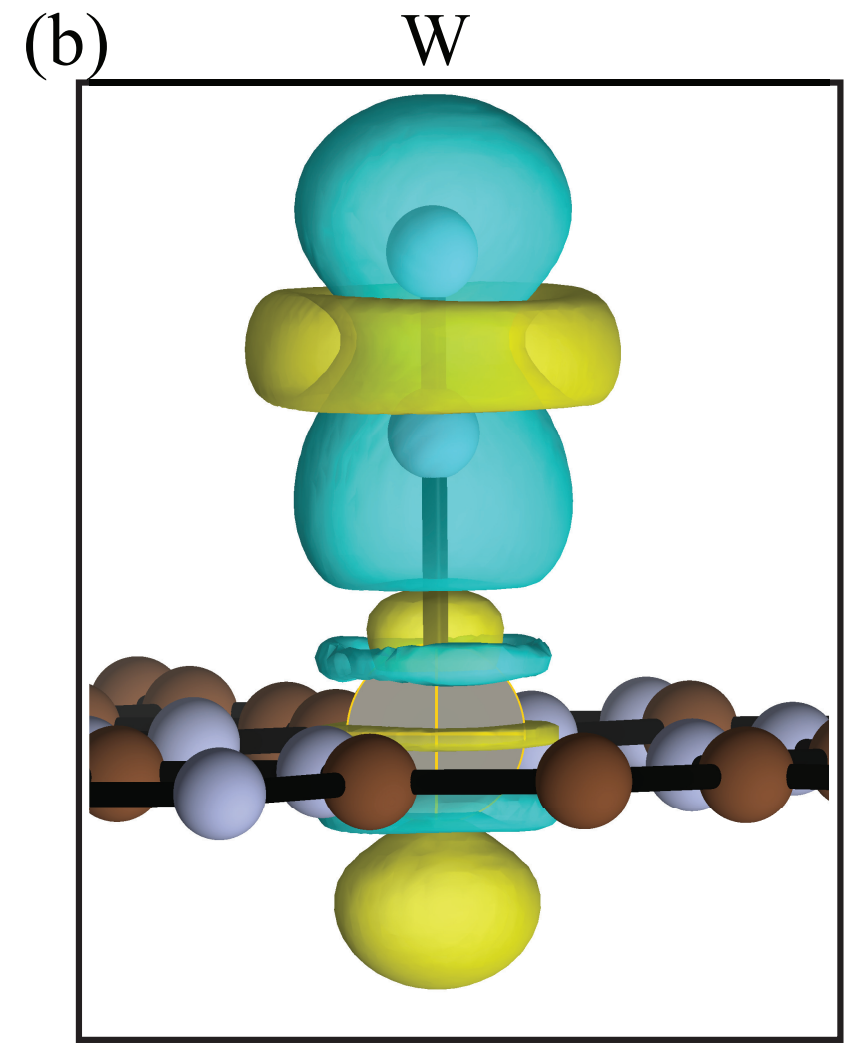

Figure S3: The charge density difference plots for $\mathrm{N}_{2}$ adsorption on (a) Mo- and (b) W-SACs, where the yellow and cyan colors represent charge depletion and accumulation, respectively. Isosurface value = $0.005 \mathrm{e} / \AA^{3}$ 

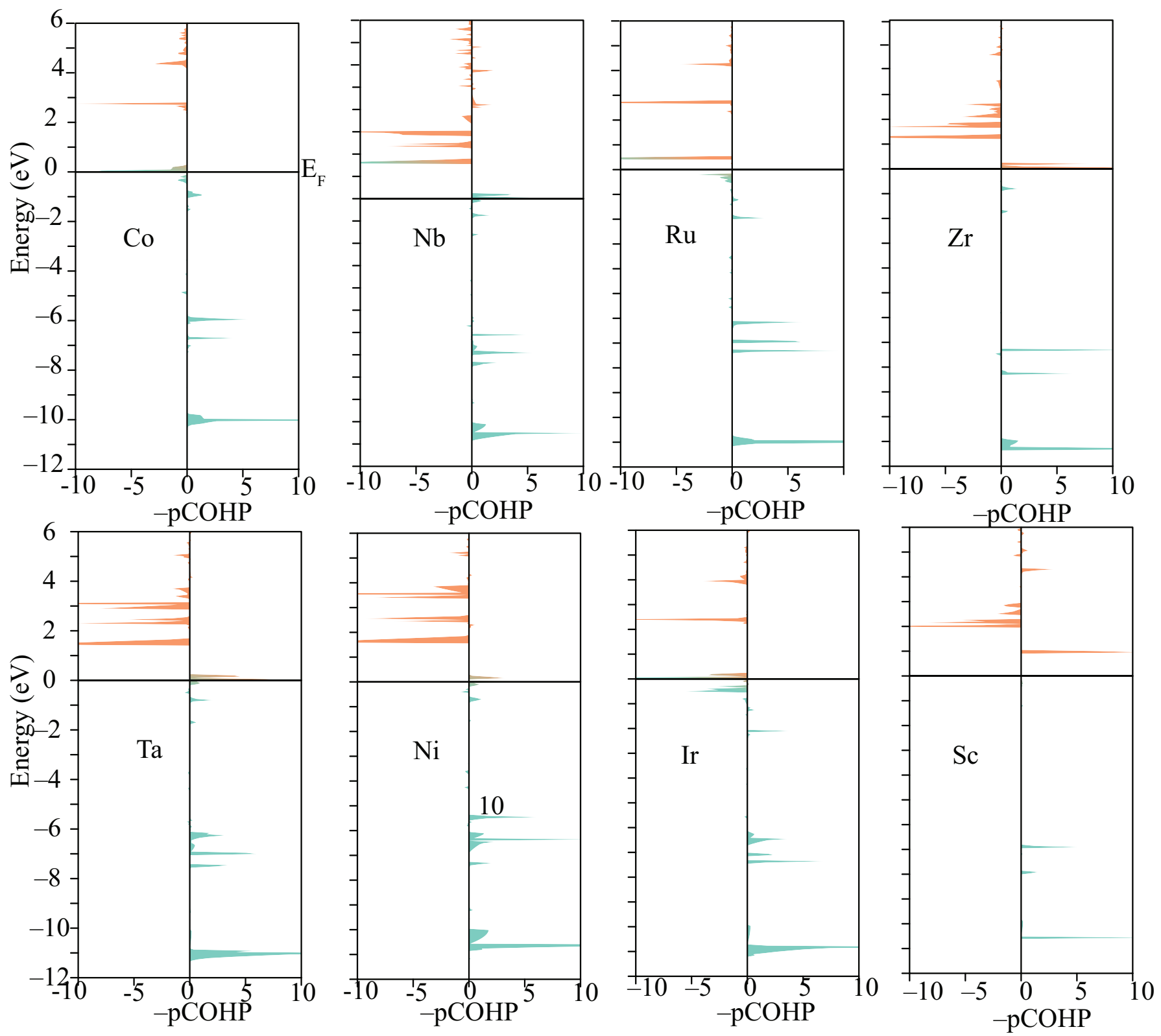

Figure S4: The -pCOHP plots for Co-, Nb-, Ru-, Zr-, Ta-, Ni-, Ir-, and Sc-SACs. 

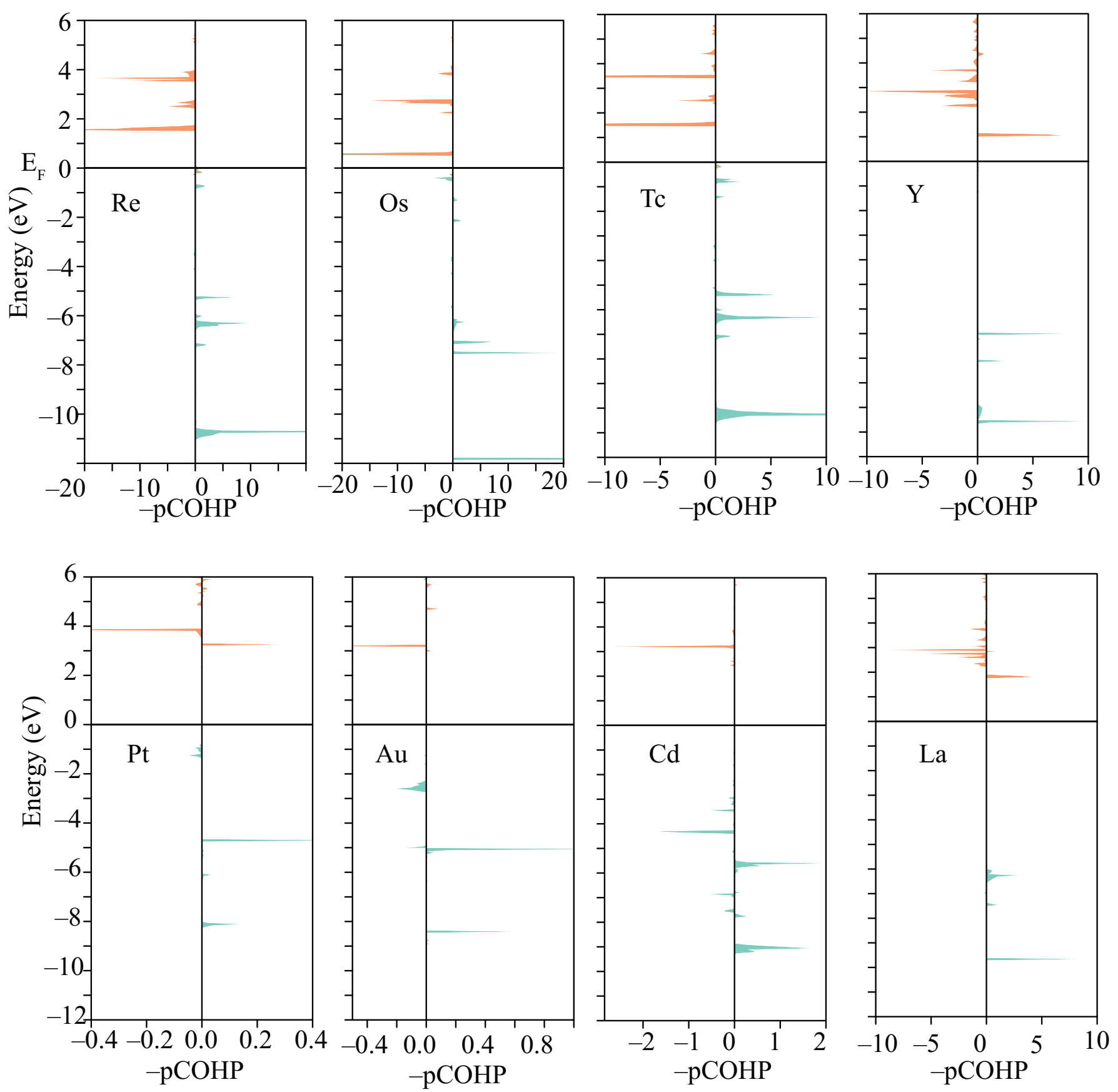

Figure S5: The -pCOHP plots for Re-, Os-, Tc-, Y-, Pt-, Au-, Cd-, and La-SACs. 

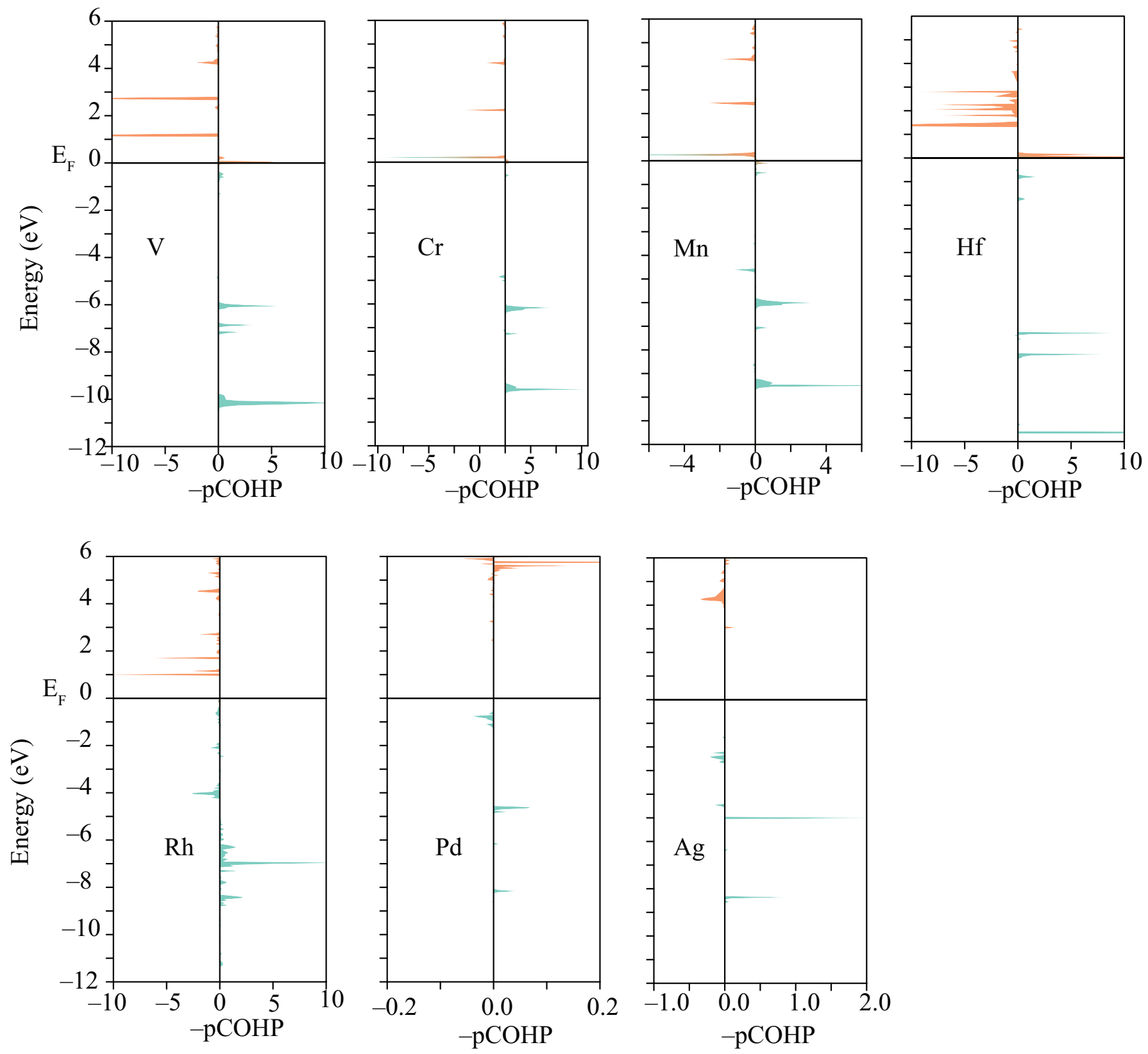

Figure S6: The -pCOHP plots for V-, Cr-, Mn-, Hf-, Rh-, Pd-, and Ag-SACs. 

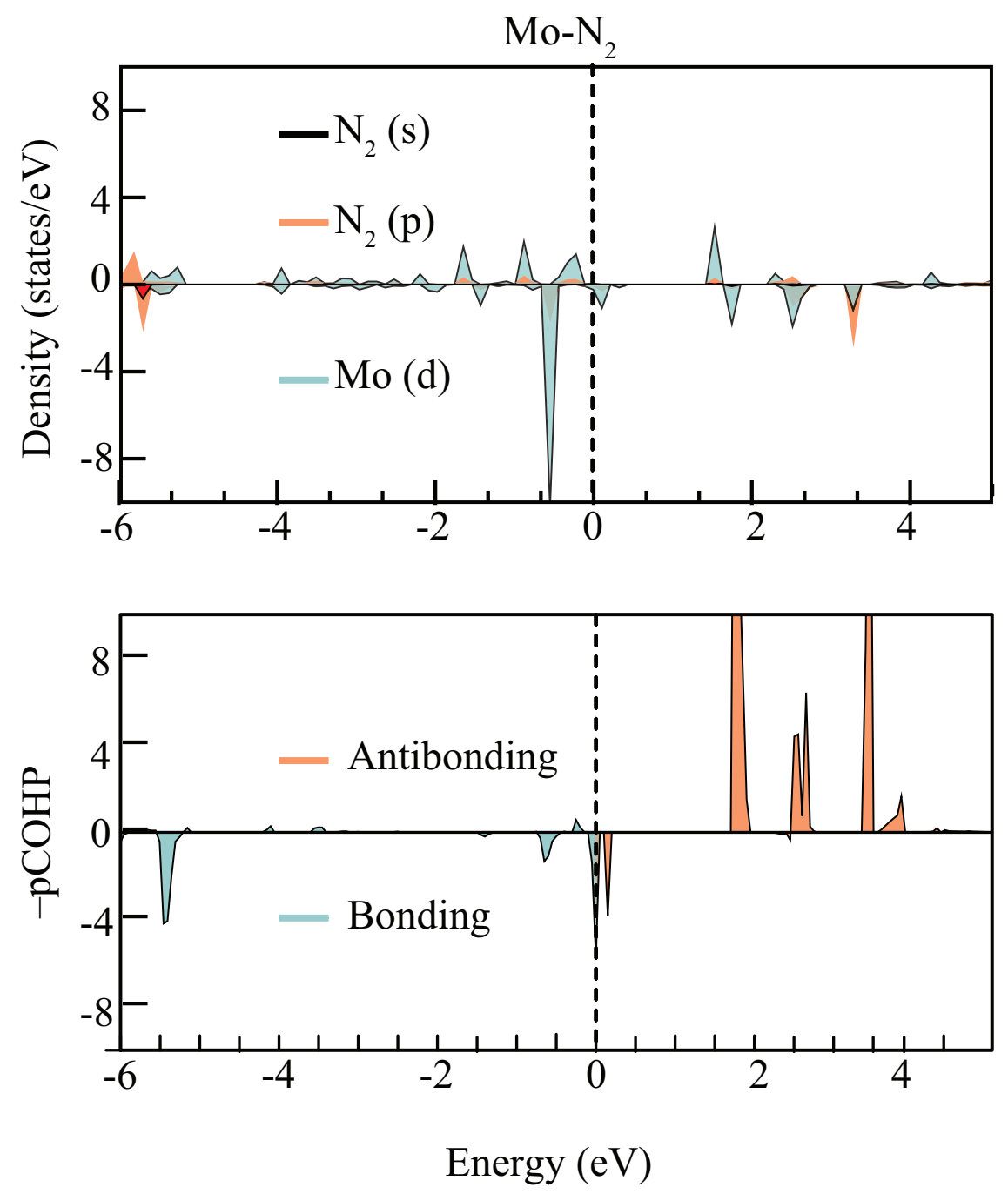

Figure S7: The pDOS for $\mathrm{N}_{2}$ adsorption on Mo-SAC and the corresponding COHP. 


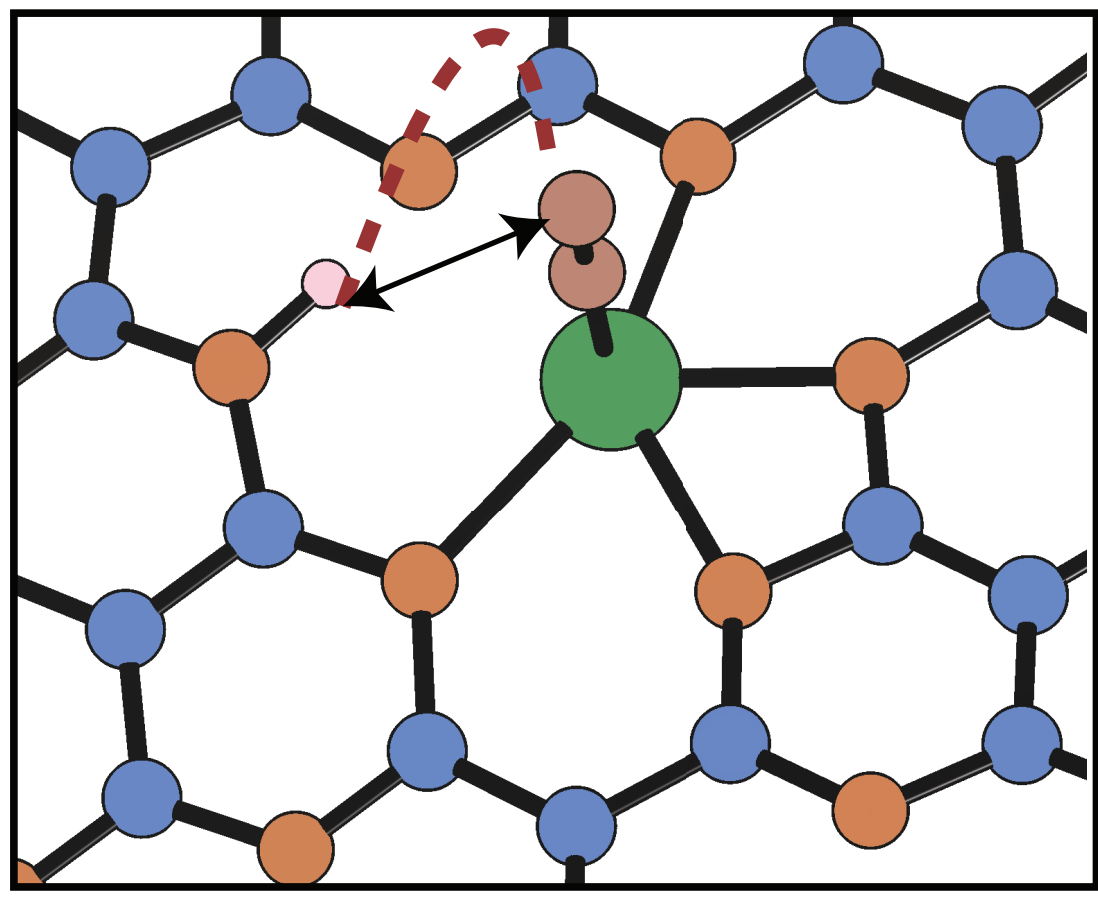

$$
\mathrm{N}-\mathrm{-H}=2.61 \AA
$$

\section{Co-adsorption}

Figure S8: The co-adsorption of $\mathrm{N}_{2}$ and $\mathrm{H}$ on the TM centers leads to the $\mathrm{H}$ intermediate preferring the support over the TMs. Blue, orange, green, pale red, and pink balls denote $\mathrm{C}, \mathrm{N}, \mathrm{TM}$, adsorbed $\mathrm{N}_{2}$, and $\mathrm{H}$, respectively. 


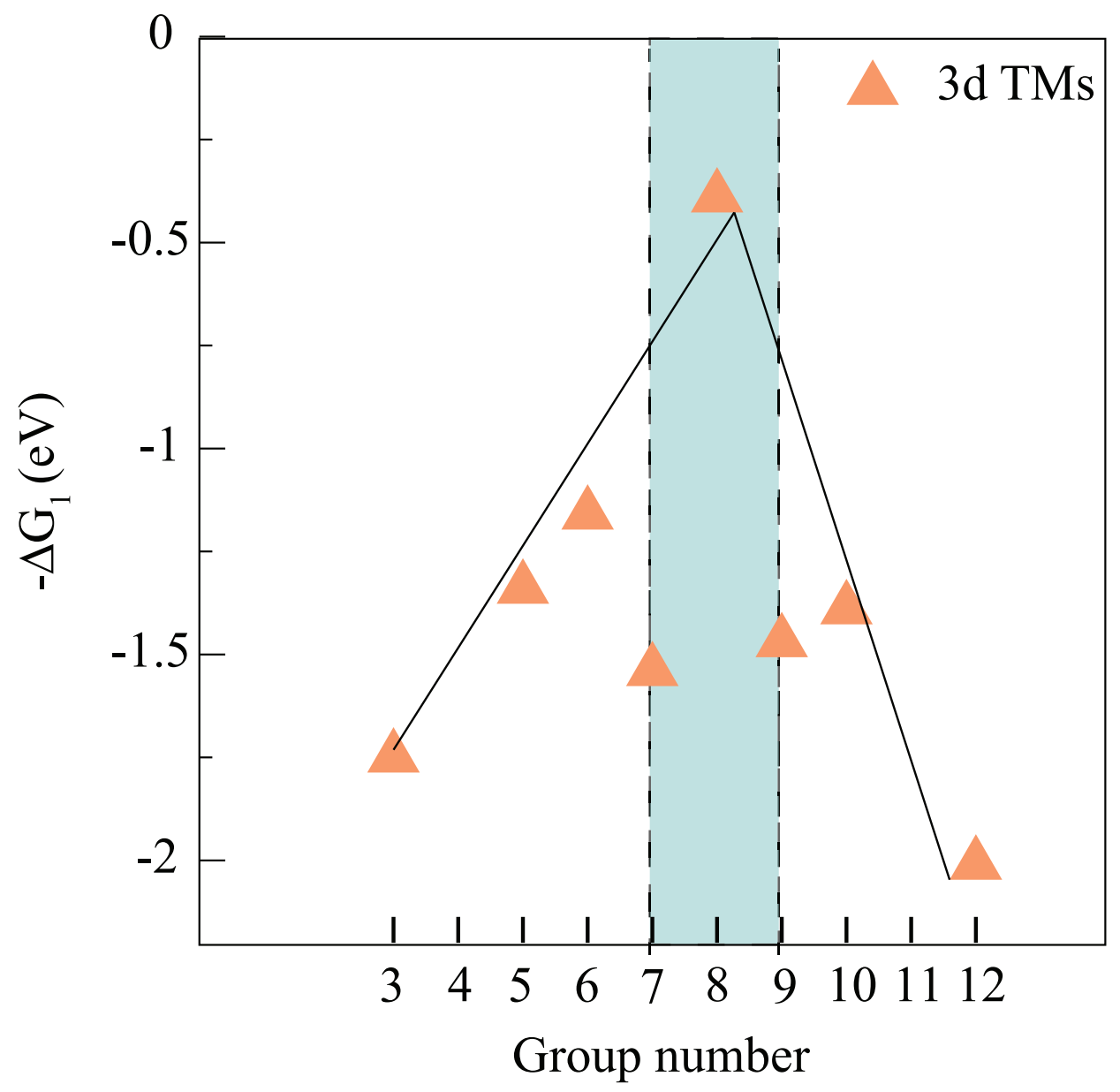

Figure S9: Variation of $\Delta \mathrm{G}_{1} *$ as a function of group number for $3 \mathrm{~d}$ metals 

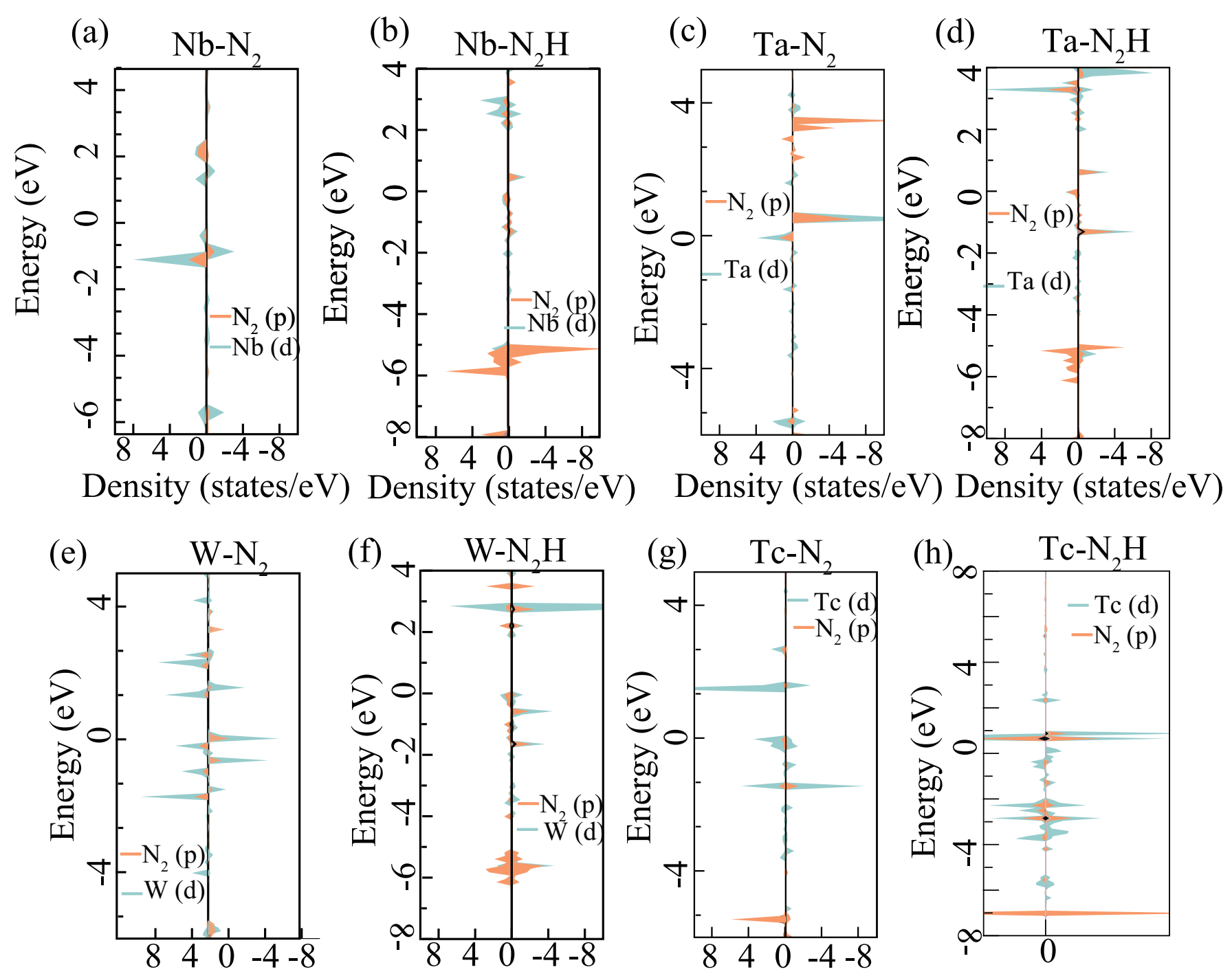

Density (states/eV) Density (states/eV) Density (states/eV) Density (states/eV)

Figure S10: The pDOS for $\mathrm{N}_{2}{ }^{*}$ and $\mathrm{N}_{2} \mathrm{H}^{*}$ intermediates adsorbed on (a,b) Nb-, (c,d) Ta-, (e,f) W-, and (g,h) Tc-SACs. 


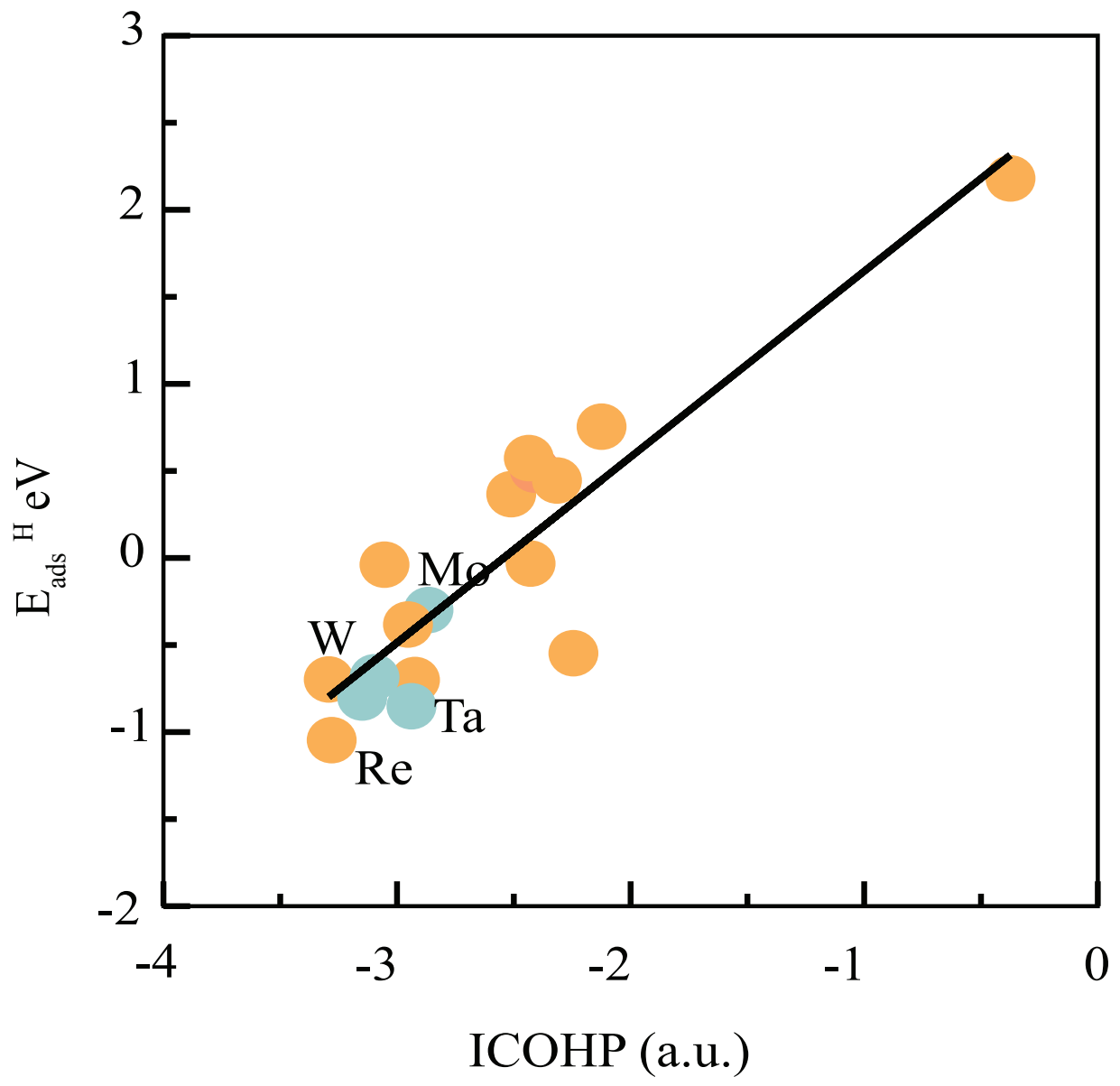

Figure S11: A linear trend between the ICOHP values for $\mathrm{H}^{*}$ adsorbed on SAC and the corresponding $\Delta \mathrm{E}_{a d s}^{H}$ values. 

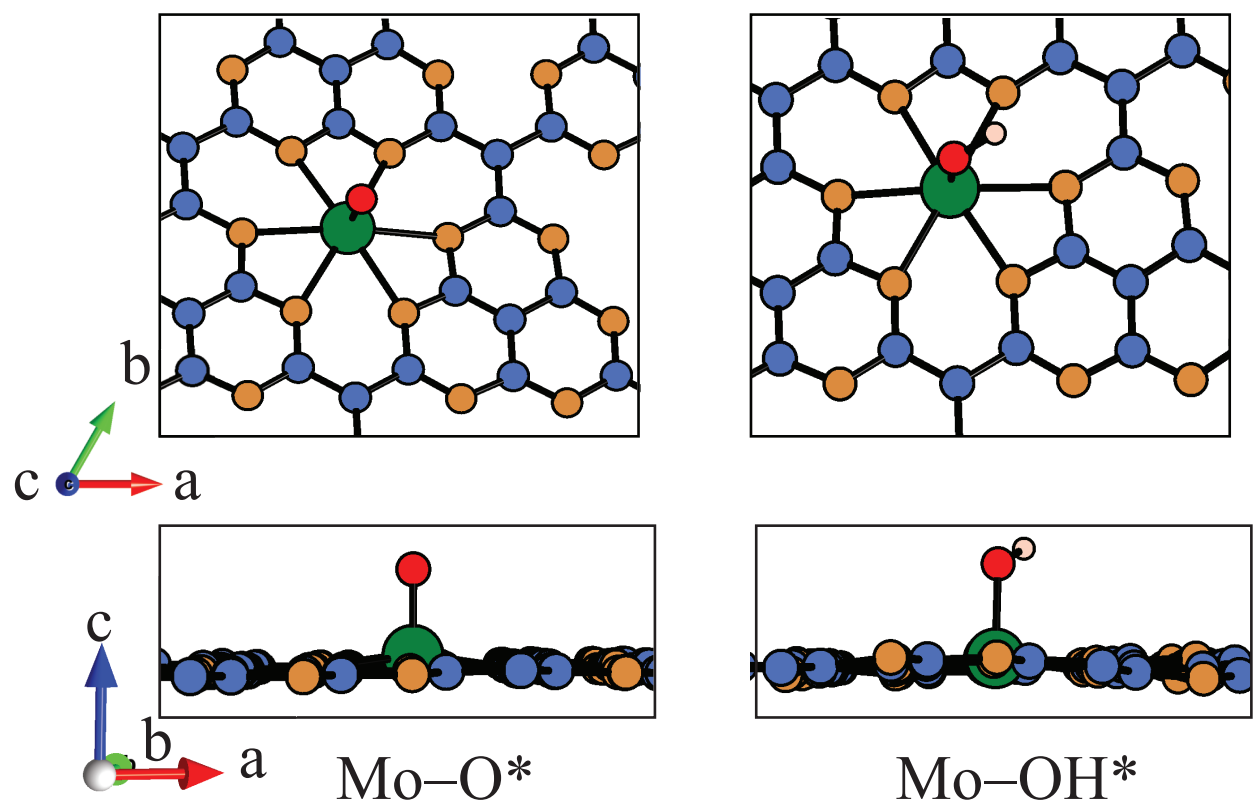

Figure S12: Optimized structure for $\mathrm{Mo}-\mathrm{O} *$ and $\mathrm{Mo}-\mathrm{OH}^{*}$ in top and side view.

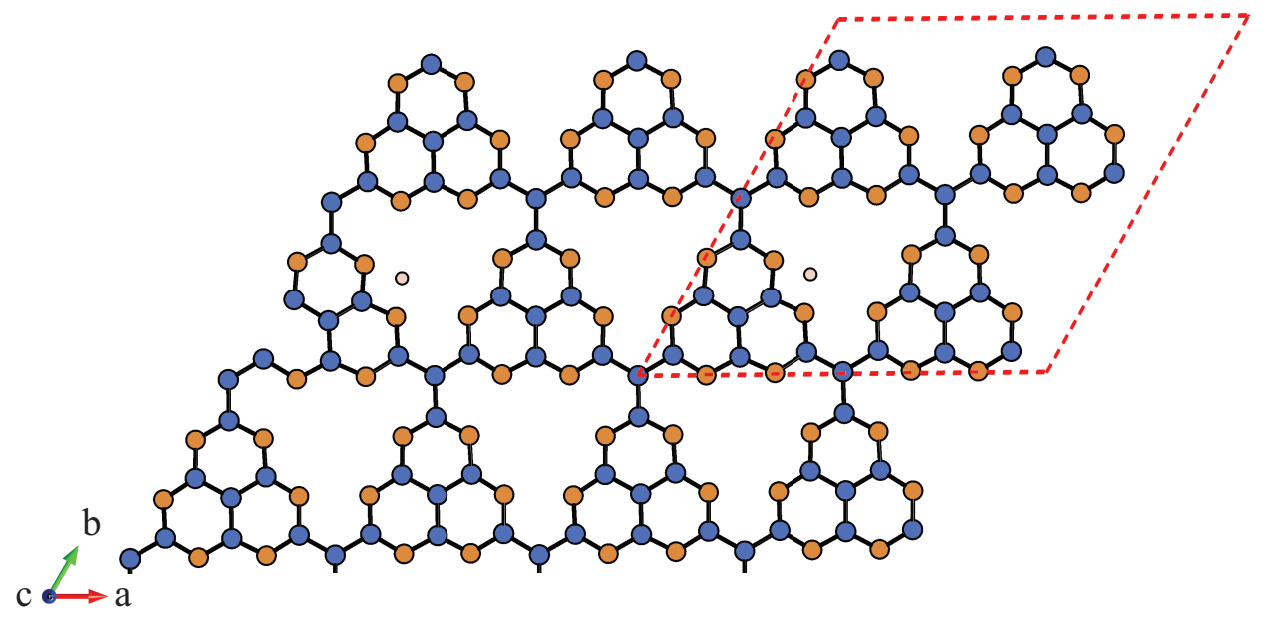

Figure S13: Optimized structure for $\mathrm{H}-\mathrm{C}_{4} \mathrm{~N}_{3}$. 
Table S1: Total energy of the system $\left(\mathrm{SAC}+\mathrm{N}_{2}\right)$, total energy of SAC without adsorbate, nitrogen Adsorption energies, ZPE -T $\Delta \mathrm{S}$, and free energies of all the TM based SACs. The reference molecule used for the calculations is $\mathrm{N}_{2}(\mathrm{~g})$

\begin{tabular}{|c|c|c|c|c|c|}
\hline Transition metal & $\begin{array}{l}\text { Total Energy } \\
\left(\mathrm{E}_{S A C+a d}\right)\end{array}$ & $\begin{array}{ll}\text { Total } & \text { Energy } \\
\left(\mathrm{E}_{S A C}\right) & \end{array}$ & $\Delta \mathrm{E}_{N_{2}}(\mathrm{eV})$ & $\mathrm{ZPE}-\mathrm{T} \Delta \mathrm{S}(\mathrm{eV})$ & $\Delta \mathrm{G}_{N_{2}}$ \\
\hline $\mathrm{Sc}$ & -503.16 & -486.08 & -0.47 & 0.09 & 0.02 \\
\hline V & -502.45 & -484.61 & -1.23 & -0.27 & -1.11 \\
\hline $\mathrm{Cr}$ & -501.82 & -485.083 & -0.13 & 0.09 & 0.35 \\
\hline Mn & -501.43 & -484.54 & -0.28 & 0.10 & 0.21 \\
\hline $\mathrm{Fe}$ & -498.77 & -482.63 & 0.45 & 0.04 & 0.89 \\
\hline Co & -498.11 & -480.76 & -0.74 & 0.10 & -0.23 \\
\hline $\mathrm{Ni}$ & -496.27 & -479.23 & -0.44 & 0.07 & 0.03 \\
\hline $\mathrm{Zn}$ & -493.16 & -476.19 & -0.36 & 0.02 & 0.05 \\
\hline $\mathrm{La}$ & -502.71 & -485.55 & -0.55 & 0.05 & -0.10 \\
\hline $\mathrm{Zr}$ & -504.81 & -487.37 & -0.83 & 0.10 & -0.32 \\
\hline $\mathrm{Nb}$ & -504.47 & -487.06 & -0.80 & 0.01 & -0.38 \\
\hline Mo & -503.15 & -485.99 & -0.55 & 0.05 & -0.10 \\
\hline $\mathrm{Tc}$ & -501.80 & -484.28 & -0.91 & 0.07 & -0.43 \\
\hline $\mathrm{Ru}$ & -499.66 & -482.28 & -0.76 & 0.04 & -0.31 \\
\hline $\mathrm{Rh}$ & -497.43 & -480.61 & 2.06 & 0.11 & 2.57 \\
\hline $\mathrm{Pd}$ & -495.58 & -478.87 & -0.10 & 0.07 & 0.37 \\
\hline $\mathrm{Ag}$ & -493.29 & -476.52 & -0.16 & -0.04 & 0.19 \\
\hline $\mathrm{Cd}$ & -493.05 & -476.15 & -0.29 & -0.03 & 0.07 \\
\hline Y & $\mid-503.98$ & -486.94 & -0.43 & 0.06 & 0.02 \\
\hline $\mathrm{Hf}$ & -506.216 & -488.68 & -0.92 & -0.04 & -0.48 \\
\hline $\mathrm{Ta}$ & -505.67 & -488.46 & -0.60 & 0.02 & -0.17 \\
\hline W & $\mid-504.86$ & -487.32 & -0.92 & 0.05 & -0.47 \\
\hline $\operatorname{Re}$ & -503.31 & -485.56 & -1.14 & 0.08 & -0.65 \\
\hline Os & -500.96 & -483.41 & -0.94 & 0.07 & -0.47 \\
\hline Ir & -498.00 & -481.42 & 0.02 & 0.04 & 0.47 \\
\hline $\mathrm{Pt}$ & -495.88 & -479.17 & -0.09 & -0.03 & 0.27 \\
\hline $\mathrm{Au}$ & -493.00 & -476.30 & -0.09 & 0.04 & 0.34 \\
\hline
\end{tabular}


Table S2: Total energy of the system $\left(\mathrm{SAC}+\mathrm{N}_{2} \mathrm{H}\right), \mathrm{N}_{2} \mathrm{H}$ Adsorption energies, ZPE -T $\Delta \mathrm{S}$, and free energies of all the TM based SACs. The reference molecule used for the calculations is $\mathrm{N}_{2}+\mathrm{H}_{2}(\mathrm{~g})$

\begin{tabular}{|l|l|l|l|l|l|}
\hline Transition metal & $\begin{array}{l}\text { Total energy } \\
\left(\mathrm{E}_{S A C+a d}\right)\end{array}$ & $\Delta \mathrm{E}_{N_{2} H}(\mathrm{eV})$ & ZPE -T $\Delta \mathrm{S}(\mathrm{eV})$ & $\Delta \mathrm{G}_{N_{2} H}$ & $\Delta \mathrm{G}_{1}$ \\
\hline $\mathrm{Sc}$ & -505.03 & 1.03 & 0.30 & 1.77 & 1.75 \\
$\mathrm{~V}$ & -505.14 & 0.54 & 0.26 & 0.23 & 1.34 \\
$\mathrm{Cr}$ & -504.35 & 0.71 & 0.30 & 1.52 & 1.16 \\
$\mathrm{Mn}$ & -503.44 & 1.08 & 0.33 & 1.81 & 1.59 \\
$\mathrm{Fe}$ & -502.05 & 0.55 & 0.31 & 1.32 & 0.42 \\
$\mathrm{Co}$ & -500.27 & 0.47 & 0.31 & 1.24 & 1.47 \\
$\mathrm{Ni}$ & -498.55 & 0.66 & 0.29 & 1.43 & 1.39 \\
$\mathrm{Zn}$ & -494.83 & 1.35 & 0.27 & 2.07 & 2.01 \\
$\mathrm{La}$ & -504.34 & 1.19 & 0.32 & 1.96 & 2.06 \\
$\mathrm{Zr}$ & -507.37 & -0.01 & 0.30 & 0.76 & 1.07 \\
$\mathrm{Nb}$ & -507.44 & -0.39 & 0.31 & 0.37 & 0.76 \\
$\mathrm{Mo}$ & -506.74 & -0.75 & 0.33 & 0.05 & 0.15 \\
$\mathrm{Tc}$ & -504.56 & -0.28 & 0.26 & 0.44 & 0.88 \\
$\mathrm{Ru}$ & -502.16 & 0.11 & 0.32 & 0.88 & 1.20 \\
$\mathrm{Rh}$ & -500.07 & 0.52 & 0.34 & 1.26 & 0.96 \\
$\mathrm{Pd}$ & -497.41 & 1.44 & 0.29 & 2.19 & 1.82 \\
$\mathrm{Cd}$ & -494.62 & 1.51 & 0.25 & 2.23 & 2.16 \\
$\mathrm{Y}$ & -505.84 & 1.09 & 0.29 & 1.86 & 1.83 \\
$\mathrm{Hf}$ & -508.90 & -0.23 & 0.23 & 0.46 & 0.94 \\
$\mathrm{Ta}$ & -509.23 & -0.78 & 0.31 & 0.00 & 0.17 \\
$\mathrm{~W}$ & -508.56 & -1.24 & 0.34 & -0.43 & 0.03 \\
$\mathrm{Re}$ & -506.46 & -0.90 & 0.28 & -0.16 & 0.49 \\
$\mathrm{Os}$ & -503.41 & 0.00 & 0.30 & 0.76 & 0.88 \\
$\mathrm{Ir}$ & -501.24 & 0.16 & 0.31 & 0.93 & 0.45 \\
$\mathrm{Pt}$ & -497.86 & 1.30 & 0.30 & 2.06 & 2.45 \\
$\mathrm{Au}$ & -494.59 & 1.69 & 0.30 & 2.48 & 2.14 \\
\hline
\end{tabular}


Table S3: Total energies of the system (SAC+adsorbates), adsorption energies, ZPE - T $\Delta \mathrm{S}$, and free energies of various intermediates for Mo-, W-, and Ta- based SACs. The reference molecule used for the calculations is $\mathrm{N}_{2}(\mathrm{~g})+\mathrm{H}_{2}(\mathrm{~g})$

\begin{tabular}{|c|c|c|c|c|c|}
\hline Transition metal & $\begin{array}{ll}\text { Total } & \text { energy } \\
\left(\mathrm{E}_{S A C+a d}\right)\end{array}$ & $\Delta \mathrm{E}_{a d s}(\mathrm{eV})$ & $\mathrm{ZPE}-\mathrm{T} \Delta \mathrm{S}(\mathrm{eV})$ & $\Delta \mathrm{G}$ & intermediate \\
\hline Mo & -510.37 & -1.00 & 0.66 & 0.19 & $\mathrm{~N}_{2} \mathrm{H}_{2} *$ \\
\hline Mo & -495.32 & -1.02 & -0.02 & -0.84 & $\mathrm{~N}^{*}$ \\
\hline Mo & -498.58 & -0.90 & 0.23 & -0.40 & $\mathrm{NH}^{*}$ \\
\hline Mo & -502.63 & -1.57 & 0.55 & -0.69 & $\mathrm{NH}_{2}{ }^{*}$ \\
\hline Mo & -506.66 & -1.12 & 0.90 & -0.53 & $\mathrm{NH}_{3} *$ \\
\hline Mo & -509.56 & -0.20 & 0.53 & 0.86 & $\mathrm{NHNH}^{*}$ \\
\hline Mo & -513.97 & -1.22 & 0.53 & 0.86 & $\mathrm{NHNH}_{2} *$ \\
\hline Mo & -517.56 & -1.43 & 1.31 & 0.53 & $\mathrm{NH}_{2} \mathrm{NH}_{2} *$ \\
\hline $\mathrm{W}$ & -512.58 & -1.88 & 0.65 & -0.70 & $\mathrm{~N}_{2} \mathrm{H}_{2}$ * \\
\hline $\mathrm{W}$ & -496.83 & -1.20 & 0.03 & -0.97 & $\mathrm{~N}^{*}$ \\
\hline $\mathrm{W}$ & -501.01 & -2.00 & 0.27 & -1.45 & $\mathrm{NH}^{*}$ \\
\hline $\mathrm{W}$ & $\mid-504.42$ & -2.02 & 0.55 & -1.14 & $\mathrm{NH}_{2} *$ \\
\hline W & -508.11 & -1.24 & 0.90 & -0.65 & $\mathrm{NH}_{3} *$ \\
\hline $\mathrm{W}$ & -511.20 & -0.50 & 0.62 & 0.64 & $\mathrm{NHNH}^{*}$ \\
\hline W & $\mid-515.83$ & -1.75 & 0.62 & 0.64 & $\mathrm{NHNH}_{2}$ \\
\hline $\mathrm{W}$ & -519.03 & -1.54 & 1.29 & 0.38 & $\mathrm{NH}_{2} \mathrm{NH}_{2} *$ \\
\hline $\mathrm{Ta}$ & -513.76 & -1.92 & 0.6 & -0.79 & $\mathrm{~N}_{2} \mathrm{H}_{2} *$ \\
\hline $\mathrm{Ta}$ & -496.83 & -0.06 & 0.03 & 0.16 & $\mathrm{~N}^{*}$ \\
\hline Та & -502.52 & -2.38 & 0.29 & -1.82 & $\mathrm{NH}^{*}$ \\
\hline $\mathrm{Ta}$ & $\mid-506.29$ & -2.76 & 0.55 & -1.86 & $\mathrm{NH}_{2} *$ \\
\hline $\mathrm{Ta}$ & $\mid-509.30$ & -1.29 & 0.80 & -0.79 & $\mathrm{NH}_{3} *$ \\
\hline $\mathrm{Ta}$ & -512.44 & -0.60 & 0.55 & 0.47 & $\mathrm{NHNH}^{*}$ \\
\hline $\mathrm{Ta}$ & $\mid-516.30$ & -1.08 & 0.55 & 0.47 & $\mathrm{NHNH}_{2} *$ \\
\hline $\mathrm{Ta}$ & $\mid-520.17$ & -1.57 & 1.30 & 0.38 & $\mathrm{NH}_{2} \mathrm{NH}_{2} *$ \\
\hline
\end{tabular}

Table S4: Total energies, ZPE, and $\mathrm{T} \Delta \mathrm{S}$ of the reference molecule.

\begin{tabular}{|c|c|c|c|}
\hline $\begin{array}{l}\text { Reference } \\
\text { molecule }\end{array}$ & $\begin{array}{ll}\text { Total } & \text { energy } \\
\left(\mathrm{E}_{r e f}\right) & \end{array}$ & $\mathrm{ZPE}(\mathrm{eV})$ & $\mathrm{T} \Delta \mathrm{S}(\mathrm{eV})$ \\
\hline $\mathrm{H}_{2}$ & -6.76 & 0.27 & 0.41 \\
\hline $\mathrm{N}_{2}$ & -16.60 & 0.15 & 0.55 \\
\hline $\mathrm{NH}_{3}$ & -19.54 & 0.91 & 0.60 \\
\hline
\end{tabular}

